\title{
Is there a role for renin profiling in selecting chronic heart failure patients for ACE inhibitor treatment?
}

\author{
P O Lim, R J MacFadyen, A D Struthers
}

\begin{abstract}
Background-It remains uncertain whether angiotensin converting enzyme (ACE) inhibitors benefit all heart failure patients or just those with renin-angiotensin-aldosterone system (RAAS) activation.

Objective-To determine whether the response to an ACE inhibitor, assessed by urine sodium excretion, was different in patients with low renin versus those with high renin.

Design-Plasma renin activity (PRA) was measured in 38 patients with stable chronic heart failure (21 male, 17 female; mean (SD) age 71 (6) years, range 59-82 years) on chronic diuretic treatment alone. They were divided into three groups: low (PRA $\leqslant 1.5 \mathrm{ng} / \mathrm{ml} / \mathrm{h}, \mathrm{n}=11$ ); normal $(1.5<\mathrm{PRA}<5, \mathrm{n}=14)$; and high (PRA $>5, \mathrm{n}=13)$. The effect of ACE inhibition was then assessed on diuretic induced natriuresis with respect to renin status.

Results-There were no significant differences in age and sex distribution between the groups. Plasma angiotensin II and aldosterone increased serially from low to high renin groups, while $24 \mathrm{~h}$ urinary sodium concentrations fell from low to high renin groups (low PRA, 96.7 (39.5); normal PRA, 90.4 (26.7); high PRA, 66.3 (18.9) mmol/1; $\mathrm{p}=0.033$ ), despite a higher diuretic dose in the high renin group. This blunted natriuretic effect of loop diuretics was caused by RAAS activation, which could partly be reversed by ACE inhibition. ACE inhibitors increased natriuresis by $22 \%$ in the high renin group ( $p=0.029)$, but had no effect in the normal and low renin groups. Within the low renin group, five of the 11 patients had persistently low renin levels despite ACE inhibition. There was a non-significant reduction in natriuresis $(-9.6 \%, \mathrm{p}=0.335)$ following ACE inhibition in this subgroup of patients.

Conclusions-About one third of heart failure patients in our study had low renin status and a non-activated RAAS, despite diuretic treatment. ACE inhibitors did not alter natriuresis significantly in this subgroup of patients, and enhanced natriuresis only in patients with high renin. There is thus tentative support for renin profiling in targeting ACE inhibitors to the most deserving, by showing that short term sodium retention does not occur in low renin patients if ACE inhibitors are withdrawn.
\end{abstract}

(Heart 2000;83:257-261)

Keywords: chronic heart failure; renin profiling; ACE inhibition; urinary sodium excretion

Over the years, the optimum treatment of chronic heart failure has been clarified by many large mortality trials. ${ }^{1-4}$ Angiotensin converting enzyme (ACE) inhibitors, $\beta$ blockers, and spironolactone have all been shown to reduce mortality. On the horizon are other promising treatments such as endothelin antagonists, angiotensin II receptor antagonists, neutral endopeptidase inhibitors, and cytokine blockers. One of the problems is that each of these new treatments further reduces the already low blood pressure of chronic heart failure, and it could soon be that a low blood pressure will become a limiting factor in treatment. This has led to the concept of individualised treatment in chronic heart failure, rather than simply adding each new treatment to the regimen when efficacy is proven. The effect of the latter could also be to limit efficacy because of reduced compliance.

Thus, instead of giving ACE inhibitors to all patients, the idea of using renin levels to select patients who are likely to respond to ACE inhibitor treatment arises. Data from the cooperative north Scandinavian enalapril survival study (CONSENSUS I) ${ }^{5}$ suggest that ACE inhibitors only improve mortality in patients with neurohormonal activation. A similar picture was seen in the vasodilator therapy of heart failure study (VeHeFT II) for high versus low renin levels. ${ }^{6}$ Therefore from these data one could argue that ACE inhibitors should only be given to those with renin-angiotensinaldosterone system (RAAS) activation, especially following diuretic treatment. ${ }^{7}$ In contrast, patients without RAAS activation may have nothing to gain from ACE inhibitor treatment ${ }^{8}$ and perhaps much to lose, in that they may still get side effects and the drugs obviously still cost money. However, withholding ACE inhibitors in heart failure patients is ethically questionable at present. It is important to determine which patients should receive ACE inhibitors and which patients can safely be withdrawn from them, as this may become an important health policy issue in the future.

Aside from mortality, morbidity is another key determinant of whether a drug should be started or withdrawn. We therefore examined whether patients with chronic heart failure who have raised renin levels are more likely to decompensate (that is, to retain sodium) in the 
short term if ACE inhibitor treatment is withdrawn or withheld. If this were the case, poor compliance with ACE inhibitors might lead to frequent hospital admissions, and "renin profiling" could help identify those individuals who are at particular risk of disease decompensation if they inadvertently stop their ACE inhibitor treatment. Extra vigilance with respect to compliance would be necessary for patients with high renin levels.

To answer these various questions, we measured renin levels during ACE inhibitor withdrawal in patients with chronic heart failure and examined the consequences of withdrawal on urinary sodium output, which obviously reflects the likelihood that heart failure will become decompensated.

\section{Methods}

PARTICIPANTS

Thirty eight consecutive patients with proven heart failure caused by ischaemic cardiomyopathy, with a left ventricular ejection fraction of less than $45 \%$ as determined by echocardiographic or radionuclide scans, were recruited from our tertiary referral heart failure centre. These were patients with compensated heart failure on optimum diuretic dosage, and all were established on ACE inhibitors. Each patient gave written consent to participate in the study, which was approved by the local Tayside medical ethics committee.

Of the 38 patients, 21 were male and 17 female. Mean (SD) age was 71 (6) years, range 59 to 82 . All patients were on ACE inhibitors and were treated with diuretics (lisinopril mean dose, 12.9 (6.1) mg; equivalent frusemide (furosemide) mean dose, 77 (34) mg at entry). None of these patients was being treated with spironolactone or $\beta$ blockers. After one week of treatment on placebo, as a substitute for ACE inhibitor treatment, they were divided into the three groups according to their PRA values: $\leqslant 1.5,1.5$ to 5 , and $>5 \mathrm{ml} / \mathrm{h} / \mathrm{min}$. The characteristics of the three groups are given in table 1 .

Table 1 Renin profiling in patients with stable chronic heart failure

\begin{tabular}{lllll}
\hline Characteristics & Low PRA & Normal PRA & High PRA & p Value \\
\hline $\mathrm{n}$ & 11 & 14 & 13 & \\
Sex (M/F) & $5 / 6$ & $6 / 8$ & $9 / 4$ & 0.333 \\
Age (years) & $73(7)$ & $71(7)$ & $71(5)$ & 0.598 \\
NYHA II/III & $3 / 8$ & $8 / 6$ & $7 / 6$ & 0.608 \\
PRA (ng/ml/h) & $0.74(0.47)$ & $2.47(0.68)$ & $11.92(6.00)$ & $<0.01$ \\
Systolic BP (mm Hg) & $135(21)$ & $127(19)$ & $129(28)$ & 0.721 \\
Diastolic BP $(\mathrm{mm} \mathrm{Hg})$ & $72(13)$ & $74(9)$ & $69(12)$ & 0.693 \\
Heart rate $($ beats/min) & $67(12)$ & $75(17)$ & $72(7)$ & 0.393 \\
Lisinopril $(\mathrm{mg}) \dagger$ & $12.7(6.1)$ & $12.9(5.4)$ & $13(7)$ & 0.99 \\
Frusemide $(\mathrm{mg})$ & $58(28)$ & $71(28)$ & $98(35)$ & 0.008 \\
Plasma sodium $(\mathrm{mmol} / \mathrm{l})$ & $134.9(4.1)$ & $135.7(3.7)$ & $134.7(3.5)$ & 0.749 \\
Plasma potassium $(\mathrm{mmol} / \mathrm{l})$ & $3.9(0.4)$ & $4.1(0.5)$ & $3.8(0.5)$ & 0.208 \\
Plasma urea $(\mathrm{mmol} / \mathrm{l})$ & $5.4(1.6)$ & $7.0(2.4)$ & $6.4(2.9)$ & 0.255 \\
Serum creatinine $(\mu \mathrm{mol} / \mathrm{l})$ & $102(21)$ & $116(39)$ & $110(32)$ & 0.565 \\
Creatinine clearance $(\mathrm{ml} / \mathrm{min}) \ddagger$ & $58(32)$ & $79(92)$ & $73(40)$ & 0.695 \\
Serum ACE $(\mathrm{U} / \mathrm{l})$ & $25.8(12.4)$ & $29.6(10.0)$ & $31.4(14.0)$ & 0.533 \\
\hline
\end{tabular}

${ }^{\star} \mathrm{p}<0.05,{ }^{\star \star} \mathrm{p}<0.01$.

†Entry dose of lisinopril, which was substituted with placebo tablets for one week before the hormonal assessments.

$\ddagger$ Calculated using the equation of [urinary creatinine concentration $\times 24$ hour urine volume]/plasma creatinine concentration.

ACE, angiotensin converting enzyme; BP, blood pressure; NYHA, New York Heart Association functional class; PRA, plasma renin activity.
INTERVENTION

The study lasted for two weeks in each patient. At entry, all patients were randomised in double blind fashion to either continue their usual ACE inhibitors or to receive an equivalent number of placebo tablets. After one week, blood samples were collected in the morning following a 30 minute period of supine rest for serum ACE activity, plasma renin activity (PRA), aldosterone, and angiotensin II. Twenty four hour urine was collected for estimation of urinary sodium concentration. All patients then crossed over to the other treatment limb for another week, after which the above assessments were repeated. Patients were advised to continue on their usual diet throughout the study.

\section{LABORATORY TESTING}

Plasma renin activity, aldosterone, and angiotensin II were measured by radioimmunoassay techniques. PRA was measured using the Biodata Renin MAIA assay (Serono Diagnostics, Woking, Surrey, UK) and plasma aldosterone using a solid phase (coated tube) radioimmunoassay technique, DPC Coat-a-Count assay (DPC, Llanberis, Caernarfon, Gwynedd, UK). Plasma angiotensin II was measured in Glasgow using Dowex $\mathrm{H}^{+}$ion exchange resin and Sep-pak C18 cartridges (Waters Associates, Milford, Massachusetts, USA) to extract angiotensin II from venous blood before assaying as described previously. ${ }^{9}$

Serum ACE activity was determined by monitoring the change in absorbance at 340 $\mathrm{nm}$ of the hydrolysis of furyl-acrylolylphenylalanyl-glycyl-glycine (FAPGG) to urylacrylolyl-phenylalanine and glycyl-glycine (Sigma-Aldrich Chemical Co, Poole, Dorset, UK) on a Roche MIRA analyser (Roche Diagnostic, Welwyn Garden City, Herts, UK). The intra-assay correlation coefficients of these measurements were all below $10 \%$.

\section{DATA ANALYSIS}

Results were expressed as mean (SD). Regression analyses were used to assess the relations between indices, and analysis of variance to assess differences between groups. Fisher's multiple comparison method was adopted in post-hoc analyses. A probability $(p)$ value of $<0.05$ was accepted as statistically significant. Statistical analyses were performed using the statistical package MINITAB $^{\circledR}$, version 12.22 .

\section{Results}

ACE INHIBITOR WITHDRAWAL

There were no significant differences in sex or age distribution across the three groups (low, normal, and high renin). Entry ACE inhibitor dosage did not differ between the groups, but there was a serial increase in diuretic dose from low to high renin groups. The baseline plasma urea, creatinine, and creatinine clearance values were not significantly different between the groups. As expected, there was an increase in plasma angiotensin II and aldosterone from low to high renin groups, despite there being no significant suppression or difference in serum ACE activity following ACE inhibitor 
Table 2 Changes in plasma angiotensin II, aldosterone, and urinary sodium concentration with and without ACE inhibition

\begin{tabular}{|c|c|c|c|}
\hline & Placebo & ACE inhibitor & Changes \\
\hline \multicolumn{4}{|c|}{ Angiotensin II (pmol/l) } \\
\hline Low PRA & $7.5(4.8)$ & $4.3(3.2)$ & 3.2 (3.7) $(95 \%$ CI 0.8 to 5.7$) ; \mathrm{p}=0.015$ \\
\hline Normal PRA & $11.4(7.7)$ & $2.74(2.3)$ & $8.7(7.0)(95 \%$ CI 4.5 to 12.9$) ; \mathrm{p}=0.001$ \\
\hline High PRA & $\begin{array}{l}49.0(46.4) \\
{ }^{\star} \mathrm{p}<0.001\end{array}$ & $\begin{array}{l}3.8(3.3) \\
\mathrm{p}=0.419\end{array}$ & $45.2(45.8)(95 \%$ CI 17.5 to 72.8$) ; \mathrm{p}=0.004$ \\
\hline \multicolumn{4}{|c|}{ Aldosterone (pmol/l) } \\
\hline Low PRA & $262(146)$ & $219(158)$ & $42(88)(95 \% \mathrm{CI}-16$ to 102$) ; \mathrm{p}=0.138$ \\
\hline Normal PRA & $336(172)$ & $234(235)$ & $102(167)(95 \%$ CI 2 to 203$) ; \mathrm{p}=0.047$ \\
\hline \multirow[t]{2}{*}{ High PRA } & $599(312)$ & $260(118)$ & 338 (281) (95\% CI 149 to 527$) ; \mathrm{p}=0.003$ \\
\hline & ${ }^{\star} \mathrm{p}<0.001$ & $\mathrm{p}=0.948$ & \\
\hline \multicolumn{4}{|c|}{ Urine sodium ( $\mathrm{mmol} / \mathrm{l})$} \\
\hline Low PRA & $96.7(39.5)$ & $101.8(33.6)$ & $-5.1(23.6)(95 \% \mathrm{CI}-21.0$ to 10.8$) ; \mathrm{p}=0.491$ \\
\hline Normal PRA & $90.4(26.7)$ & $94.5(33.1)$ & $-4.1(24.0)(95 \% \mathrm{CI}-18.6$ to 10.4$) ; \mathrm{p}=0.551$ \\
\hline \multirow[t]{2}{*}{ High PRA } & $66.3(18.9)$ & $80.8(22.5)$ & $-14.6(21.3)(95 \%$ CI -27.4 to -1.73$) ; \mathrm{p}=0.02$ \\
\hline & ${ }^{\star} \mathrm{p}=0.033$ & $\mathrm{p}=0.231$ & \\
\hline
\end{tabular}

*Post hoc pairwise comparisons suggested that the plasma angiotensin II and aldosterone levels were not different between the low and normal PRA groups, but the levels of these hormones were significantly higher in the high PRA groups than in either low or normal PRA groups.

ACE, angiotensin converting enzyme; PRA, plasma renin activity.

Table 3 Correlation coefficients of RAAS hormones, diuretic doses, and urinary sodium excretion following withdrawal of ACE inhibitors

\begin{tabular}{lrrrr}
\hline$(n=38)$ & \multicolumn{1}{l}{ PRA } & Angiotensin II & Aldosterone & Frusemide \\
\hline Angiotensin II & $0.515 ; \mathrm{p}=0.001$ & & & \\
Aldosterone & $0.498 ; \mathrm{p}=0.002$ & $0.679 ; \mathrm{p}<0.001$ & & \\
Frusemide & $0.286 ; \mathrm{p}=0.082$ & $0.273 ; \mathrm{p}=0.098$ & $0.255 ; \mathrm{p}=0.134$ & $-0.346 ; \mathrm{p}=0.034$ \\
24 h urine sodium excretion & $-0.302 ; \mathrm{p}=0.066$ & $-0.344 ; \mathrm{p}=0.035$ & $-0.621 ; \mathrm{p}<0.001$ & -0.34 \\
\hline
\end{tabular}

ACE, angiotensin converting enzyme; PRA, plasma renin activity; RAAS, renin-angiotensin-aldosterone system.

withdrawal. There was also a fall in urinary sodium concentrations from low to high renin groups despite the higher diuretic doses used (table 2).

There were correlations between PRA, angiotensin II, and aldosterone (table 3, fig 1), suggesting that high renin status following diuretic treatment was associated with RAAS activation. There were, however, no significant correlations between the diuretic dose and the neurohormones. Natriuretic efficacy was blunted at higher diuretic doses with chronic diuretic treatment, and 24 hour urine sodium excretion was also negatively correlated with plasma aldosterone.

EFFECTS OF ACE INHIBITION

After adding ACE inhibitors for one week, there was no significant difference in blood pressure reduction between the three groups. There was, however, enhanced natriuresis in the high renin group (table 2). The urinary sodium concentration rose by a mean of $22 \%(p=0.029)$ in the high renin group, without significant changes in the normal and low renin groups. Despite ACE

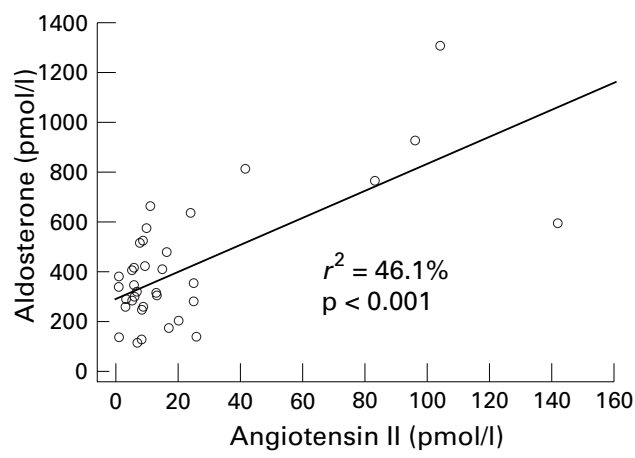

Figure 1 Correlation between plasma angiotensin II and aldosterone levels with ACE inhibitors withdrawn. inhibition, the trend for lower urinary sodium concentrations from high to low renin status remained unchanged between the three groups. ACE inhibition was more effective in reducing angiotensin II and aldosterone levels in the high renin group, but the resulting absolute levels of these hormones were not different between the groups (table 2). The serum ACE activity was similarly suppressed across the renin groups with ACE inhibition (14.1 (5.4), 16.1 (14.9), and 10.8 (3.1) $\mathrm{U} / \mathrm{l}, \mathrm{p}=0.411)$. The change in angiotensin II and aldosterone with ACE inhibition correlated well in the whole cohort $(r=0.56, \mathrm{p}<0.001)$, whereas the correlation between urinary sodium concentrations and the diuretic doses strengthened $(r=-0.46$, $\mathrm{p}=0.004)$. The regression slopes between these two latter indices did not, however, differ significantly according to whether or not there was ACE inhibition. Stratifying the patients into three groups by diuretic dosage $-<40 \mathrm{mg}$ ( $\mathrm{n}=13), 40-80 \mathrm{mg}(\mathrm{n}=17)$, and $>80 \mathrm{mg}$ $(\mathrm{n}=7)-$ did not predict the natriuretic response to ACE inhibition.

Within the low renin subgroup, five of the 11 patients continued to have low renin levels after the addition of ACE inhibitors. This represented $13 \%$ of the total study population. There was a non-significant reduction in urinary sodium concentration $(-9.6 \%$, $\mathrm{p}=0.335)$ following $\mathrm{ACE}$ inhibition in this subgroup of patients. The rise of PRA in the remaining six subjects in the low renin group was not associated with an alteration in the natriuretic efficacy of the diuretics.

\section{Discussion}

While renin profiling has been advocated in hypertension, ${ }^{10}{ }^{11}$ it has not so far been used in clinical practice in managing patients with chronic heart failure, despite the fact that the 
RAAS is of central importance in the pathophysiology of heart failure. Patients with activation of the RAAS have a worse prognosis $^{5}$ but respond better to ACE inhibition. ${ }^{5}$ Whether ACE inhibition confers advantages on heart failure patients without RAAS activation is less certain.

We assessed the renin profile of our patients during chronic treatment with diuretics. In this way we could determine the degree of RAAS activation in individual patients, which has prognostic value. ${ }^{56}$ An intriguing finding in our study is that up to one third of the patients had low renin levels, and of these half retained their low levels even when ACE inhibitors were added for a week. Conversely, about one third of our patients had RAAS activation following chronic diuretic treatment. The diuretic induced rise in PRA generated an increase in circulating angiotensin II. Angiotensin II, a powerful stimulator of aldosterone secretion from the adrenal cortex, causes a rise in plasma aldosterone. In turn, aldosterone acts on the distal renal tubules, inhibiting sodium excretion. This may explain why higher diuretic doses in chronic treatment were associated with a blunted natriuretic efficacy, as a result of diuretic induced RAAS activation in susceptible patients. The group of patients with high renin responded to ACE inhibition with enhanced natriuresis and a greater reduction in angiotensin II and aldosterone, thus counteracting the increased aldosterone related distal renal tubular sodium reabsorption. In contrast, ACE inhibition had little effect in promoting natriuresis in diuretic treated patients with normal or low baseline renin. In the absence of activated RAAS, especially in the low renin group, the baseline low levels of these hormones mean that there is less room for suppression-hence the lack of the added natriuretic effect seen in the high renin group.

In a separate study, ACE inhibition in heart failure patients without RAAS activation was not associated with any beneficial alteration in haemodynamic status, which was, however, improved by calcium channel blockade. ${ }^{12}$ Hence there is a whole range of ACE inhibitor effects that now appear to occur only in patients with RAAS activation, including haemodynamic effects, effects on urinary sodium, effects on neurohormones, and even effects on mortality. ${ }^{568}$

Although it is intriguing that a sizeable subgroup of our chronic heart failure patients had low levels of renin despite chronic diuretic treatment, ${ }^{12}$ it was even more surprising that low renin often persisted in the presence of ACE inhibitor treatment. This emphasises the heterogeneity of the RAAS in patients with chronic heart failure. Such heterogeneity must be taken into account before one can even consider the idea of using renin profiling to individualise ACE inhibitor treatment. Our study is clearly only one step in this direction and much more work is required before renin profiling could be used in practice to individualise ACE inhibitor treatment. The work described here does give reassurance that, at least in the short term, ACE inhibitor with- drawal only causes sodium retention in those with high renin levels and not in those with low renin levels. Previous data from the CONSENSUS I trial give additional reassuring information on mortality by showing that even the grade IV patients in that trial had little to gain from ACE inhibitor treatment in the short term, at least if they did not have neuroendocrine activation.

These data are also relevant to the question of how often decompensation and hospital readmission of patients with chronic heart failure can be attributed to non-compliance with treatment. One of the great unknowns in chronic heart failure is why some patients decompensate frequently and others do not. Non-compliance is thought to be an important contributing factor, although most commentators consider that the key factor is nonadherence with diuretics. These data show that decompensation can occur even if compliance with diuretic treatment is good. They show that non-compliance with an ACE inhibitor can itself cause sodium retention, even when compliance with diuretic treatment is good, but that this only occurs to any extent in those with high renin levels. Therefore patients with chronic heart failure on ACE inhibitor treatment are in a precarious position and should be advised not to allow their ACE inhibitor treatment to lapse as they run a high risk of retaining sodium even if they continue with diuretics.

Not surprisingly, angiotensin II reactivation occurs mainly in patients with high circulating concentrations of renin and angiotensin I. One could argue that angiotensin II reactivators should be selected to receive a higher dose of ACE inhibitor (in this study, the average lisinopril dose was only $13 \mathrm{mg} /$ day). Thus a further use of renin profiling might be to select particular patients for higher than average ACE inhibitor dosage. It could be argued that if ACE inhibitors are prescribed in patients with low renin levels, these patients should not be subjected to large, hypotension producing doses, which should be reserved for those with high renin levels.

Finally, the finding that the favourable natriuretic enhancing effects of ACE inhibitors were only apparent in the high renin group does not itself imply that ACE inhibitors should not be prescribed to patients with low renin status. More research is clearly required. This study takes us one step towards the idea that renin profiling may help to select patients with chronic heart failure for ACE inhibitor treatment.

\section{CONCLUSIONS}

About one third of the patients with heart failure in our study had low renin status with a non-activated RAAS despite diuretic treatment. ACE inhibitors did not alter natriuresis in this subgroup of patients but significantly enhanced natriuresis in patients with high renin levels following chronic diuretic treatment. We acknowledge the help of our patients in the completion of
this project and to Sister Jessamine Robson for her invaluable this project and to Sister Jessamine Robson for her invaluable
help in practical data collection. We thank the British Heart help in practical data collection.
Foundation for financial support. 
1 The cardiac insufficiency bisoprolol study II (CIBIS-II): a randomised trial. Lancet 1999;353:9-13.

2 Effect of enapril on mortality and the developm heart failure in asymptomatic patients with reduced left ventricular ejection fractions. The SOLVD Investigators. $N$ Engl F Med 1992;327:685-91.

3 Effect of ramipril on mortality and morbidity of survivors of acute myocardial infarction with clinical evidence of heart failure. The Acute Infarction Ramipril Efficacy (AIRE) Study Investigators. Lancet 1993;342:821-8.

4 Pitt D. ACE inhibitor co-therapy in patients with heart failure: rationale for the randomized aldactone evaluation study (RALES). Eur Heart $\mathcal{F}$ 1995;16(suppl N): 107-10.

5 Swedberg K, Eneroth P, Kjekshus J, et al. Effects of enalapril and neuroendocrine activation on prognosis in severe congestive heart failure (follow-up of the CONSENSUS trial). CONSENSUS Trial Study Group. Am f Cardiol 1990;66: 40-4D.

6 Francis GS, Cohn JN, Johnson G, et al. Plasma norepinephrine, plasma renin activity, and congestive heart failure. phrine, plasma renin activity, and congestive heart failure. II. The V-HeFT VA Cooperative Studies Group. Circulation 1993;87:VI40-8.
7 Francis GS, Benedict C, Johnstone DE, et al. Comparison of neuroendocrine activation in patients with left ventricular dysfunction with and without congestive heart failure. A substudy of the Studies of Left Ventricular Dysfunction (SOLVD). Circulation 1990;82: $1724-9$

8 Lee WH, Packer M. Prognostic importance of serum sodium concentration and its modification by convertingenzyme inhibition in patients with severe chronic heart failure. Circulation 1986;73:257-67.

9 Morton JJ, Webb DJ. Measurement of plasma angiotensin II [letter]. Clin Sci 1985;68:483-4.

$10 \mathrm{Laragh} \mathrm{JH}$. A decade of angiotensin-converting enzyme (ACE) inhibition. Am f Med 1992;92:3-7S.

11 Lim PO, Rodgers P, Cardale K, et al. Potentially high prevalence of primary aldosteronism in a primary-care population. Lancet 1999;353:40.

12 Cody RJ, Laragh JH, Atlas SA, et al. Converting enzyme inhibition to identify and treat renin-mediated or sodiumvolume related forms of increased peripheral resistance in hypertension and in congestive heart failure. $f$ Hypertens Suppl 1983;1:77-84.

\section{Perivalvar abscess of the mitral valve annulus with perforation owing to infective endocarditis}

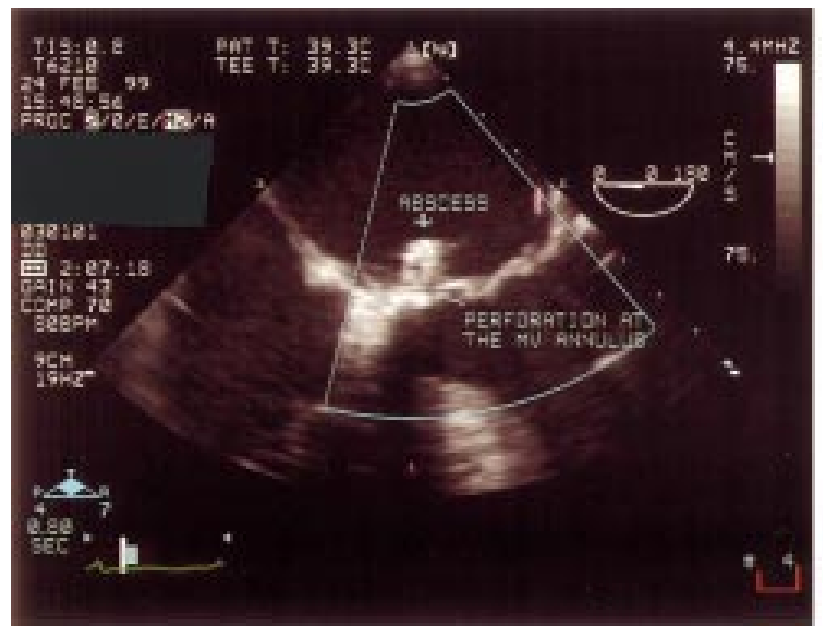

A 74 year old man presented with unstable angina and moderate aortic stenosis. He had an aortic valve replacement (Carpentier Edwards $19 \mathrm{~mm}$ porcine valve) and three reversed saphenous vein grafts to the first obtuse marginal, left anterior descending, and right coronary arteries. He was referred back three months later with a two week history of tiredness, night sweats, and weight loss of $5 \mathrm{~kg}$. There was no history of any dental work or other surgical procedures after his cardiac operation. His temperature was $38.2^{\circ} \mathrm{C}$ and there were no peripheral stigmata of infective endocarditis.

Cardiac examination revealed a pansystolic murmur in the mitral area with a soft ejection murmur in the aortic area. Laboratory investigations showed microscopic haematuria,

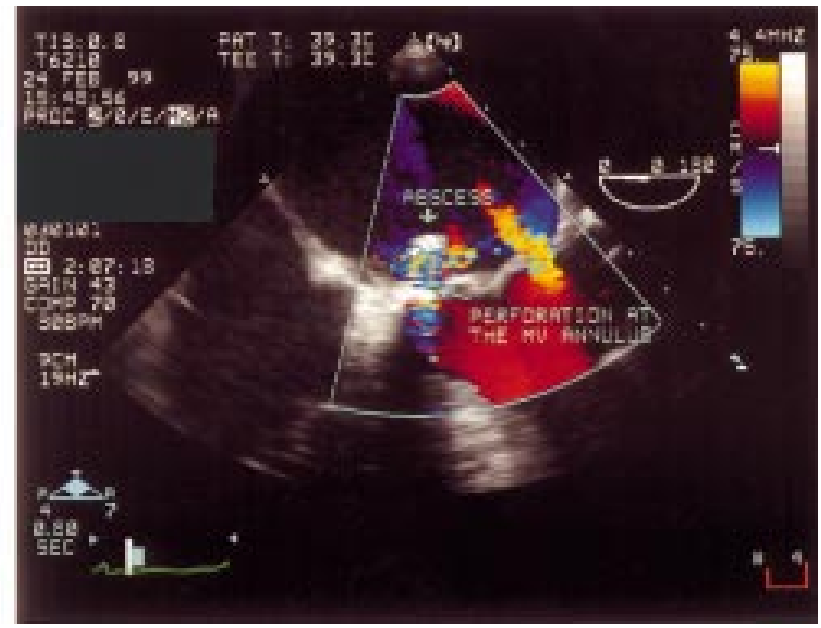

haemoglobin $97 \mathrm{~g} / 1$, white cell count $14 \times 10^{9} / 1$, and an erythrocyte sedimentation rate of $83 \mathrm{~mm}$ in the first hour. ECG showed second degree heart block (Wenckebach) with left bundle branch block pattern that was not present on previous ECGs. One of three sets of blood cultures grew Streptococcus bovis. The images on transoesophageal echocardiography were particularly striking. There was a perivalvar abscess of the mitral valve annulus extending from the anteromedial to the posteromedial part of the mitral valve (left) and colour flow Doppler showed perforation of the mitral valve annulus (right).

JACOB EASAW MAGDIE EL-OMAR MARK RAMSEY 